\title{
Pravastatin and endothelium dependent vasomotion after coronary angioplasty: the PREFACE trial
}

H J G H Mulder, M J Schalij, B Kauer, R F Visser, P R M van Dijkman, J W Jukema, A $\mathrm{H}$ Zwinderman, A V G Bruschke

\begin{abstract}
Objective-To test the hypothesis that the 3-hydroxy-3-methylglutaryl coenzyme-A reductase inhibitor pravastatin ameliorates endothelium mediated responses of dilated coronary segments: the PREFACE (pravastatin related effects following angioplasty on coronary endothelium) trial.

Design-A double blind, randomised, placebo controlled, multicentre study.

Setting-Four hospitals in the Netherlands.

Patients-63 non-smoking, non-hypercholesterolaemic patients scheduled for elective balloon angioplasty (pravastatin 34, placebo 29).

Interventions-The effects of three months of pravastatin treatment ( $40 \mathrm{mg}$ daily) on endothelium dependent vasomotor function were studied. Balloon angioplasty was undertaken one month after randomisation, and coronary vasomotor function tests using acetylcholine were performed two months after balloon angioplasty. The angiograms were analysed quantitatively.

Main outcome measures-The efficacy measure was the acetylcholine induced change in mean arterial diameter, determined in the dilated segment and in an angiographically normal segment of an adjacent non-manipulated coronary artery.

Results-Increasing acetylcholine doses produced vasoconstriction in the dilated segments $(\mathrm{p}=0.004)$ but not in the normal segments. Pravastatin did not affect the vascular response to acetylcholine in either the dilated segments $(p=0.09)$ or the non-dilated sites. Endothelium dependent vasomotion in normal segments was correlated with that in dilated segments $(r=0.47, \mathrm{p}<0.001)$. There were fewer procedure related events in the pravastatin group than in the placebo group $(\mathrm{p}<0.05)$.

Conclusions-Endothelium dependent vasomotion in normal segments is correlated with that in dilated segments. A significant beneficial effect of pravastatin on endothelial function could not be shown, but in the dilated segments there was a trend towards a beneficial treatment effect in the pravastatin group.
\end{abstract}

(Heart 2001;86:533-539)

Keywords: angioplasty; endothelium; acetylcholine; pravastatin

Department of Cardiology, Leiden

University Medical

Centre, Albinusdreef

2, PO Box 9600, 2300

RC Leiden,

Netherlands

H J G H Mulder

M J Schalij

J W Jukema

A V G Bruschke

Department of

Medical Statistics,

Leiden University

A $\mathrm{H}$ Zwinderman

St Elisabeth Hospital, Leiderdorp,

Netherlands

B Kauer

St Joseph Hospital,

Veldhoven,

Netherlands

R F Visser

Bronovo Hospital, The Hague, Netherlands

P R M van Dijkman

Correspondence to:

Dr Schalij

m.j.schalij@lumc.nl

Accepted 25 July 2001
Restenosis after percutaneous transluminal coronary angioplasty (PTCA) is still a major limitation and it occurs in $30-50 \%$ of the patients within six months of the procedure. ${ }^{1}$ Many efforts have been made to reduce this complication, including treatment with 3-hydroxy-3-methylglutaryl coenzyme A (HMG-CoA) reductase inhibitors. ${ }^{2}$ These drugs have been shown to be effective in the primary and secondary prevention of cardiovascular events; however, there is disagreement about their influence on the restenosis process. ${ }^{3-5}$ As well as reducing plasma lipids, HMG-CoA reductase inhibitors have many pleiotropic effects, including positive effects on endothelial function, that may contribute to their clinical success. ${ }^{67}$ In the complex process of restenosis an important role for the endothelium has been implicated. ${ }^{8}$ The endothelium in previously dilated segments has regrown after traumatic endothelial denudation and it has diminished functional capacity. ${ }^{10}$ This so called regenerated endothelium may partially recover after PTCA and, like native endothelium, appears to be susceptible to cardiovascular risk factors. ${ }^{11-15}$

Few studies have been performed to investigate endothelial function in vivo in humans after PTCA, and there have been no randomised trials examining the influence of strong risk modifiers such as HMG-CoA reductase inhibitors. This is the reason why we designed the PREFACE study (pravastatin related effects following angioplasty on coronary endothelium).

\section{Methods}

STUDY DESIGN AND SUBJECTS

PREFACE was a randomised, double blind, placebo controlled, multicentre study. The protocol was approved by the ethics review board of each of the four participating centres, and written informed consent was obtained from all the patients. The inclusion criteria were the presence of single or two vessel coronary artery disease $(>50 \%$ diameter stenosis) requiring PTCA of one stenosis in the left anterior descending or circumflex coronary artery. Exclusion criteria were as follows:

- age $>70$ years or $<30$ years

- total serum cholesterol $>7.0 \mathrm{mmol} / \mathrm{l}$ $(273 \mathrm{mg} / \mathrm{dl})$ or $<4.0 \mathrm{mmol} / 1(156 \mathrm{mg} / \mathrm{dl})$

- uncontrolled hypertension

- current smoking 
Table 1 Baseline characteristics

\begin{tabular}{llll}
\hline Characteristic & $\begin{array}{l}\text { Pravastatin group } \\
(n=34)\end{array}$ & $\begin{array}{l}\text { Placebo group } \\
(n=36)\end{array}$ & $p$ Value \\
\hline Age (years) & $56.3(1.6)$ & $57.2(1.3)$ & NS \\
Sex (M/F) & $27 / 7$ & $31 / 5$ & NS \\
Smoking history & $28(82 \%)$ & $31(86 \%)$ & NS \\
Previous consumption (n/day) & $15.3(2.5)$ & $19.5(2.6)$ & NS \\
Duration of smoking (years) & $27.5(2.6)$ & $29.8(11.1)$ & NS \\
History of MI & $7(21 \%)$ & $14(39 \%)$ & NS \\
History of PTCA/CABG & $0(0 \%)$ & $0(0 \%)$ & NS \\
History of CVA & $1(3 \%)$ & $1(3 \%)$ & NS \\
History of hypertension & $10(29 \%)$ & $11(30 \%)$ & NS \\
Diabetes & $1(3 \%)$ & $5(14 \%)$ & NS \\
Vessel to be dilated (LAD/Cx) & $25 / 9$ & $21 / 15$ & NS \\
\hline
\end{tabular}

Values are mean (SEM) or $\mathrm{n}(\%)$

$\mathrm{CABG}$, coronary artery by pass graft; $\mathrm{Cx}$, circumflex coronary artery; CVA, cerebrovascular accident; $\mathrm{LAD}$, left anterior descending coronary artery; $\mathrm{M} / \mathrm{F}$, male/female; MI, myocardial infarction; PTCA, percutaneous transluminal coronary angioplasty.

Table 2 Non-procedural events

\begin{tabular}{llll}
\hline Events & Pravastatin group $(n=34)$ & Placebo group $(n=36)$ & $p$ Value \\
\hline Death & 0 & 0 & \\
Myocardial infarction & 1 & 0 & \\
CABG & 0 & 1 & \\
Repeat PTCA & 3 & 4 & \\
Repeat PTCA+CABG & 1 & 0 & NS \\
Event-free n(\%) & $29(85 \%)$ & $31(86 \%)$ & \\
\hline
\end{tabular}

- previous revascularisation procedure of the vessel to be dilated

- severe hepatic disease

- unstable angina pectoris

- left bundle branch block

- bifascicular block

- earlier treatment with an HMG-CoA reductase inhibitor drug.

The patients were randomly assigned to pravastatin (40 mg once daily) or placebo. Standard balloon angioplasty was performed after one month. Patients requiring stent placement during this procedure were excluded from the angiographic follow up but remained in the study for clinical follow up. Recatheterisations for endothelial function tests were performed three months after randomisation (two months after PTCA). A final clinical evaluation was performed 30 weeks after randomisation.

Procedure related events were defined as events occurring during or within 48 hours of the PTCA procedure. Events were monitored for a period of 30 weeks after inclusion.

\section{ASSESSMENT OF ENDOTHELIUM DEPENDENT}

\section{VASOMOTOR FUNCTION}

Coronary endothelium dependent vasomotion was assessed by an established protocol, initially used by Ludmer and colleagues. ${ }^{16}$ All vasoactive drugs were discontinued 24 hours before the study. A temporary pacing catheter was positioned in the right ventricular apex in stand-by mode to avoid acetylcholine induced bradycardia. After the administration of 5000 IU of heparin, a 6 French guiding catheter (ACS-Guidant, Minneapolis, Minnesota, USA) was positioned in the ostium of the left coronary artery. After the target segments were identified, baseline angiograms before and after a two minute saline infusion were obtained. Thereafter, endothelium dependent vasomotion was assessed by serial two minute intracoronary infusions of acetylcholine (Dispersa AG, Winterthur, Switzerland), with the final intracoronary concentrations estimated to be $10^{-8}, 10^{-7}$, and $10^{-6} \mathrm{M}$ (assuming a flow rate of $80 \mathrm{ml} / \mathrm{min}$ ). ${ }^{16}$ After the acetylcholine infusion, endothelium independent vasomotion was assessed by infusion of glyceryl trinitrate at $40 \mu \mathrm{g} / \mathrm{min}$ for two minutes. The infusates were given through the guiding catheter using an infusion pump.

Within 15 seconds before each infusion, standardised single plane coronary arteriography was done using a non-ionic contrast medium (Iopamiro) and a syringe pump ( $9 \mathrm{ml}$, $7 \mathrm{ml} / \mathrm{s}$ ). Before each infusion, the catheter was filled with a predetermined volume of infusate for administration into the left main coronary artery at the beginning of the infusion. At the end of each infusion, the guiding catheter was aspirated to avoid bolus administration of acetylcholine. Finally, angiography was done again after a bolus injection of glyceryl trinitrate, with views identical to those used during the angioplasty procedure. Arterial blood pressure, heart rate, and ECG were monitored continuously.

We chose not to use an infusion catheter because we wanted to study two segments in, respectively, the left circumflex coronary artery and the left anterior descending coronary artery. The use of an infusion catheter would have directed the infusion preferentially towards one or the other artery. We also hypothesised that the introduction of adjuvant catheters into the coronary artery could of itself introduce bias. We standardised the procedures carefully to minimise any bias.

\section{QUANTITATIVE CORONARY ANGIOGRAPHY AND} DATA ANALYSIS

Quantitative coronary angiography was undertaken in a blinded fashion at the Heartcore angiographic laboratory (Leiden, Netherlands) using the cardiovascular measurement system CMS V2.5 (Medis, Leiden, The Netherlands). ${ }^{17}$ Target segments for analysis were the dilated segment and an angiographically normal segment. The latter was defined as an angiographically smooth walled coronary artery segment in a coronary artery adjacent to the dilated coronary artery (for example, a segment from the left anterior descending coronary artery when angioplasty was performed in the circumflex branch). The lumen data for these segments were recorded from single plane end diastolic frames obtained before and after each infusion. Segment responses were calculated as the percentage change in the mean segment diameter after the infusion relative to the mean segment diameter at baseline.

EXERCISE STRESS TESTING

At baseline and follow up, standardised multistage submaximal ( $85 \%$ of the predicted maximum workload) upright bicycle stress tests were performed. The test was considered positive if the following criteria were met:

- if there was a $>0.10 \mathrm{mV}$ horizontal or downsloping ST segment displacement

- if severe angina or limiting dyspnoea developed 
Table 3 Quantitative coronary angiography results at PTCA and follow up

\begin{tabular}{llll}
\hline Characteristic & $\begin{array}{l}\text { Pravastatin } \\
\text { group }(n=34)\end{array}$ & $\begin{array}{l}\text { Placebo group } \\
(n=30)\end{array}$ & $p$ Value \\
\hline Predilatation & $2.30(0.08)$ & $2.33(0.08)$ & NS \\
MSD (mm) & $1.09(0.06)$ & $1.12(0.06)$ & NS \\
MLD (mm) & $2.71(0.09)$ & $2.71(0.10)$ & NS \\
RefD (mm) & & & \\
Postdilatation & & & NS \\
MSD (mm) & $2.56(0.07)$ & $2.61(0.08)$ & NS \\
MLD (mm) & $1.91(0.07)$ & $1.97(0.07)$ & NS \\
RefD (mm) & $2.78(0.08)$ & $2.83(0.09)$ & \\
& & & NS \\
Follow up & & & NS \\
MSD (mm) & $2.49(0.07)$ & $2.58(0.09)$ & \\
MLD (mm) & $1.73(0.08)$ & $1.83(0.08)$ & \\
RefD (mm) & $2.77(0.08)$ & $2.80(0.10)$ & NS \\
Net gain & & & NS \\
MSD (mm) & $0.19(0.05)$ & $0.28(0.09)$ & NS \\
MLD (mm) & $0.63(0.07)$ & $0.73(0.09)$ & \\
\hline
\end{tabular}

Values are mean (SEM).

MLD, minimum lumen diameter; MSD, mean segmen diameter; RefD, reference diameter.

- if systolic blood pressure fell by more than $20 \mathrm{~mm} \mathrm{Hg}$

- if significant arrhythmias were induced.

The $85 \%$ predicted workload criterion was chosen to ensure that patients would also be able to reach this workload at baseline (before PTCA).

\section{STATISTICAL ANALYSIS}

For sample size calculation the following assumptions were made about mean segment diameter changes: a between-treatment group difference of $14 \%$, a standard deviation of $19 \%$, a power of 0.80 , and an overall $\alpha$ of 0.05 . This resulted in 62 analysable subjects. The primary efficacy measure was the acetylcholine induced change in arterial diameter relative to the baseline value. Differences between baseline clinical characteristics of the two groups were compared using $\chi^{2}$ tests for the noncontinuous variables and independent Student's $t$ tests for continuous variables. Dose responses to acetylcholine at individual infusate dosages were compared using Student's $t$ tests. Serial changes of data were analysed by analysis of variance (ANOVA) and analysis of covariance (ANCOVA) with repeated measures by use of the MIXED procedure of SAS. The baseline mean segment diameter served as a covariate. Linear regression analysis was performed using the change in the dilated segment as the dependent variable. All analyses were two tailed, and a value of $\mathrm{p}<0.05$ was considered to be significant. Results are presented as mean (SEM).

\section{Results}

PATIENTS AND BASELINE CHARACTERISTICS

Between 1996 and 1999, 72 eligible patients scheduled for elective balloon angioplasty were randomised to receive pravastatin (35) or placebo (37). Two patients, one in each group, were excluded from the study within days after randomisation because of protocol violation (serum cholesterol $>7 \mathrm{mmol} / \mathrm{l}$ ). Baseline characteristics of the treatment groups were similar (table 1).

\section{EVENT ANALYSIS}

Procedure related events were only observed in the placebo group $(p<0.05)$. Six bail out stent procedures had to be performed because of serious dissections obstructing flow after PTCA. Events not related to the procedure were equally distributed between the treatment groups (table 2). Repeat PTCA was the most common event. There was no mortality.

Table 4 Vasomotor endothelial function testing

\begin{tabular}{|c|c|c|c|}
\hline & Pravastatin group & Placebo group & $p$ Value \\
\hline Dilated segment & $(n=34)$ & $(n=29)$ & \\
\hline \multicolumn{4}{|l|}{ Lumen (maximum dilatation with GTN) } \\
\hline Segment length (mm) & $14.42(0.96)$ & $14.98(0.90)$ & 0.68 \\
\hline $\operatorname{MSD}(\mathrm{mm})$ & $2.42(0.09)$ & $2.54(0.10)$ & 0.42 \\
\hline $\operatorname{MLD}(\mathrm{mm})$ & $1.78(0.09)$ & $1.83(0.58)$ & 0.71 \\
\hline$\% \mathrm{DS}(\%)$ & $30.6(2.5)$ & $32.1(2.6)$ & 0.68 \\
\hline \multicolumn{4}{|l|}{ Baseline } \\
\hline $\operatorname{MSD}(\mathrm{mm})$ & $2.22(0.09)$ & $2.34(0.11)$ & 0.41 \\
\hline $\operatorname{MLD}(\mathrm{mm})$ & $1.56(0.09)$ & $1.70(0.11)$ & 0.32 \\
\hline \multicolumn{4}{|l|}{ Endothelial function } \\
\hline \multicolumn{4}{|l|}{ Response relative to baseline (\%) } \\
\hline $10^{-8} \mathrm{~mol} / 1$ & $-1.20(1.09)$ & $-2.25(0.88)$ & 0.46 \\
\hline $10^{-7} \mathrm{~mol} / 1$ & $-2.38(1.14)$ & $-5.01(1.99)$ & 0.25 \\
\hline $10^{-6} \mathrm{~mol} / 1$ & $-4.80(1.29)$ & $-7.46(2.09)$ & 0.28 \\
\hline Normal segment & $(\mathrm{n}=30)$ & $(\mathrm{n}=27)$ & \\
\hline \multicolumn{4}{|l|}{ Lumen (maximum dilatation with GTN) } \\
\hline Segment length ( $\mathrm{mm})$ & $10.11(0.60)$ & $12.26(0.74)$ & 0.03 \\
\hline $\operatorname{MSD}(\mathrm{mm})$ & $2.96(0.11)$ & $3.02(0.14)$ & 0.51 \\
\hline $\operatorname{MLD}(\mathrm{mm})$ & $2.64(0.12)$ & $2.66(0.13)$ & 0.80 \\
\hline$\% \mathrm{DS}$ & $9.4(1.0)$ & $11.1(1.1)$ & 0.26 \\
\hline \multicolumn{4}{|l|}{ Baseline } \\
\hline $\operatorname{MSD}(\mathrm{mm})$ & $2.53(0.11)$ & $2.68(0.14)$ & 0.39 \\
\hline $\operatorname{MLD}(\mathrm{mm})$ & $2.27(0.11)$ & $2.36(0.13)$ & 0.59 \\
\hline \multicolumn{4}{|l|}{ Endothelial function } \\
\hline \multicolumn{4}{|l|}{ Response relative to baseline (\%) } \\
\hline $10^{-8} \mathrm{~mol} / 1$ & $-3.4(1.4)$ & $-3.5(1.7)$ & 0.95 \\
\hline $10^{-7} \mathrm{~mol} / 1$ & $-4.4(1.5)$ & $-3.7(1.6)$ & 0.75 \\
\hline $10^{-6} \mathrm{~mol} / 1$ & $-5.2(2.0)$ & $-7.0(2.3)$ & 0.56 \\
\hline
\end{tabular}

Values are mean (SEM). Negative values indicate vasoconstriction.

GTN: glyceryl trinitrate infusion, single plane analysis; MLD, minimum lumen diameter; MSD, mean segment diameter; \%DS, percentage diameter stenosis. 

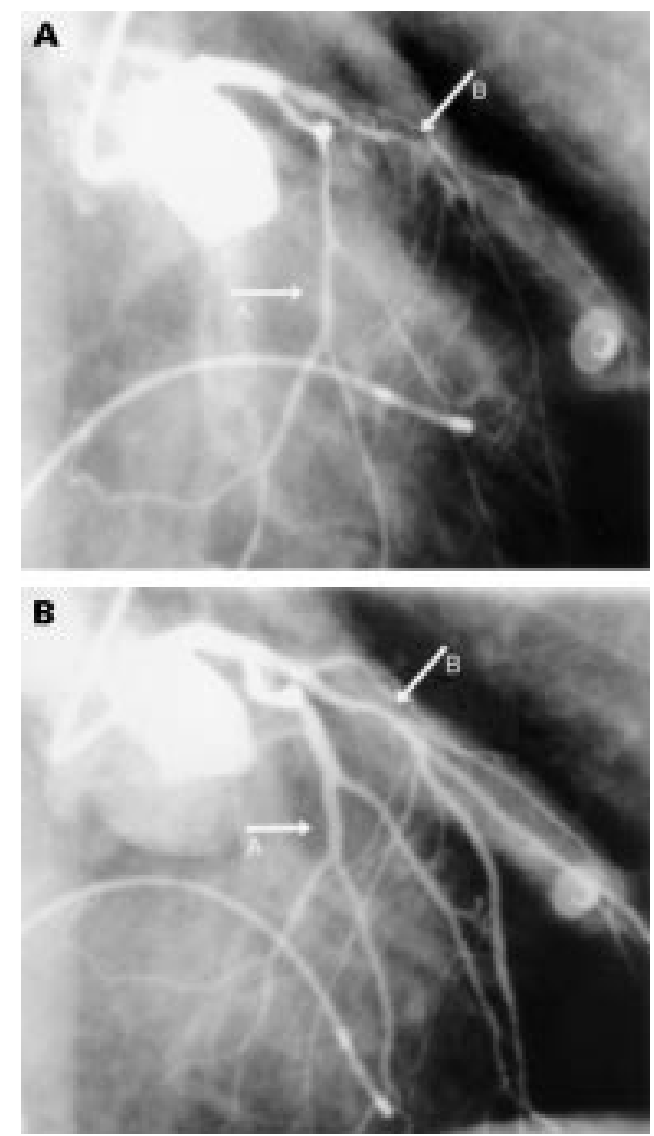

Figure 1 Examples of angiograms. (A) Example of an angiogram obtained after infusion of acetylcholine, in this case causing severe vasoconstriction. (B) The same coronary tree after infusion of glyceryl trinitrate. A, normal segment; $B$, dilated segment.

PTCA PROCEDURE

Seventy patients underwent PTCA. Six where excluded from angiographic analysis and further angiographic follow up because of bail out stent placement. The observed changes were similar between the two groups (table 3 ).

\section{ENDOTHELIUM DEPENDENT VASOMOTOR}

FUNCTION

One patient withdrew for personal reasons before angiographic follow up (placebo group). The acetylcholine challenge was therefore confined to 63 patients ( $90 \%$ follow up; pravastatin group 34 patients, placebo group 29 patients) (table 4). No complications were observed during the tests.

The lumen measurements in the dilated and normal segments at baseline and after glyceryl trinitrate infusion where similar in the two treatment groups. The only exception was the length of the normal segment, which was slightly greater in the placebo group. At baseline the dilated segments showed vasoconstriction (relative to glyceryl trinitrate infusion) of $8.3 \%$ in the pravastatin group and $7.8 \%$ in the placebo group (NS); the normal segments showed vasoconstriction of $14.5 \%$ in the pravastatin group and $11.3 \%$ in the placebo group (NS). Intracoronary infusion of saline did not change the arterial diameters.

Acetylcholine infusion evoked vasodilatation in some patients, but vasoconstriction prevailed.

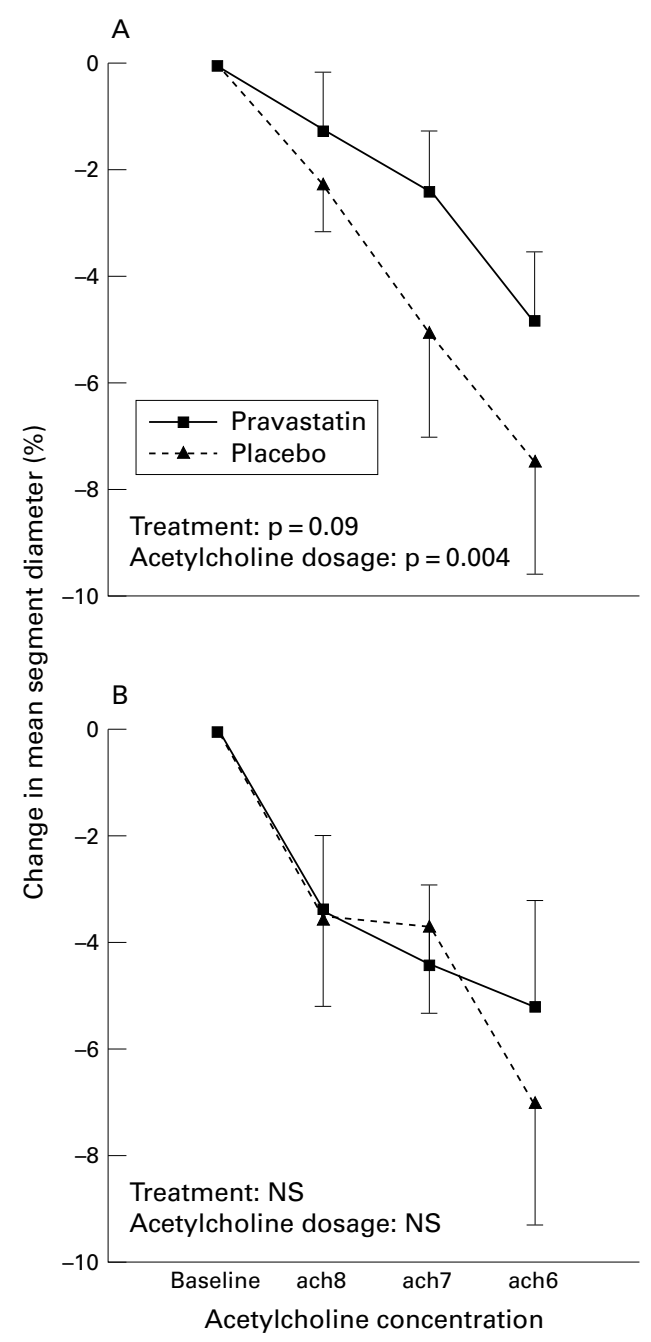

Figure 2 (A) Endothelium dependent vessel diameter changes in previously dilated coronary artery segments. There is an increase in vasoconstriction with infusion of higher concentrations of acetylcholine. There is no significant difference between the treatment groups. (B) Endothelium dependent vessel diameter changes in an angiographically normal segment. There is no significant increase in vasoconstriction with infusion of higher concentrations of acetylcholine. There is also no significant difference between the treatment groups. ach8/7/6, estimated intracoronary acetylcholine concentrations of $10^{-8}, 10^{-7}$, and $10^{-6} \mathrm{M}$

The dilated segments showed increasing degrees of vasoconstriction with increasing concentrations of acetylcholine infused $(p=0.004)$ (fig 1). A trend was found towards less vasoconstriction in the dilated segments in the pravastatin group than in the placebo group ( $p=0.09)$. The normal segments did not show significantly increased constriction at higher acetylcholine dosages (fig 2). There was no difference in vasomotion between the two treatment groups. Acetylcholine evoked responses in dilated segments were correlated with those in normal segments $(r=0.47, \mathrm{p}<0.001)$. The dilated segments had a stronger vasoconstrictor response than the normal segments.

SERUM LIPIDS

At the time of endothelial function testing, the low density lipoprotein cholesterol concentration had fallen by $36 \%$ compared with baseline 
Table 5 Lipid concentrations at baseline and at the time of endothelial function testing

\begin{tabular}{llll}
\hline Characteristic & $\begin{array}{l}\text { Pravastatin } \\
\text { group }\end{array}$ & Placebo group & p Value \\
\hline Baseline & $5.90(0.14)$ & $5.97(0.14)$ & $\mathrm{NS}$ \\
Total cholesterol & $1.20(0.05)$ & $1.21(0.05)$ & $\mathrm{NS}$ \\
HDL & $3.80(0.16)$ & $4.01(0.14)$ & $\mathrm{NS}$ \\
LDL & $2.15(0.22)$ & $1.72(0.12)$ & $\mathrm{NS}$ \\
Triglycerides & & & \\
Endothelial function testing & $4.47(0.10)$ & $5.87(0.13)$ & $<0.001$ \\
Total cholesterol & $1.34(0.08)$ & $1.23(0.05)$ & $\mathrm{NS}$ \\
HDL & $2.42(0.11)$ & $3.79(0.13)$ & $<0.001$ \\
LDL & $1.62(0.18)$ & $1.82(0.18)$ & $\mathrm{NS}$ \\
Triglycerides & $7.45(0.48)$ & $6.86(0.13)$ & $\mathrm{NS}$ \\
White blood cell count $\left(10^{9}\right)$ & $245(5)$ & $242(5)$ & $\mathrm{NS}$ \\
Platelet count $\left(10^{9}\right)$ & & & \\
Change from baseline to endothelial function testing & $-1.43(0.12)$ & $-0.11(0.10)$ & $<0.001$ \\
Total cholesterol & $0.15(0.06)$ & $0.01(0.04)$ & $<0.05$ \\
HDL & $-1.26(0.16)$ & $-0.18(0.09)$ & $<0.001$ \\
LDL & $-0.47(0.11)$ & $-0.11(0.11)$ & $<0.001$ \\
Triglycerides & & & \\
\hline Values arem & & &
\end{tabular}

Values are mean (SEM), mmol/1.

HDL, high density lipoprotein; LDL, low density lipoprotein.

in the pravastatin group (table 5). Total serum cholesterol had fallen by $24 \%$ and high density serum cholesterol had increased by $12 \%$. In the placebo group the lipid concentrations remained unchanged. There was no association between acetylcholine evoked reactions and lipid concentrations, either as absolute values at time of the acetylcholine challenge or as relative changes between challenge and baseline.

\section{Discussion}

As well as having an effect on serum cholesterol, HMG-CoA reductase inhibitors may improve endothelial function. ${ }^{9}$ In a substudy of an earlier published trial by our group (REGRESS: regression growth evaluation statin study) we assessed restenosis after two years of pravastatin treatment and showed that this had a significant positive effect on the restenosis process. ${ }^{3}{ }^{17}$ In the present study we therefore tried to clarify the effect of pravastatin treatment on the recovery of endothelial function after PTCA.

Acetylcholine evoked vasomotion reflects the endothelial function of coronary arteries. ${ }^{16}$ The observed luminal changes are the net result of opposing effects of acetylcholine on the coronary artery wall - that is, a direct tone increasing effect on the smooth muscle cell layer and a nitric oxide mediated tone decreasing effect acting through the endothelial cells. The resulting smooth muscle tone determines the magnitude and direction of the vessel size change, along with factors such as arterial wall elasticity and arterial blood pressure.

After angioplasty the vascular wall properties change, while the regrown endothelium (neoendothelium) also differs from the native endothelium. ${ }^{18}$ Animal models suggest that neoendothelium is functionally compromised. ${ }^{11}$ In keeping with this, we found an association between vessel size changes in normal and dilated segments after acetylcholine challenge, with the dilated segments reacting in a more compromised (vasoconstrictive) way than the normal segments. Furthermore we found a significant increase in vasoconstrictor effect with increasing doses of acetylcholine in the dilated segment. These results imply a dynamic postdilatation coronary segment and diminished endothelial function of the dilated segment relative to the normal segment.

While there are other studies showing hypersensitivity in dilated coronary segments, ${ }^{19}$ there have only been two reports of endothelial function in dilated coronary arteries in human subjects in vivo using serial acetylcholine infusions and quantitative coronary angiography. ${ }^{20} 21$ The results of these small observational studies were contradictory as regards the ability of the dilated segment to react to acetylcholine.

The clinical success of HMG-CoA reductase inhibitors is thought to be caused in part by their positive effect on endothelial function. ${ }^{72}$ The effects of statins on endothelial function in native coronary arteries have been studied by Treasure and colleagues and Anderson and associates, ${ }^{23}{ }^{24}$ and the acetylcholine evoked changes in our pravastatin treated normal coronary artery segments were similar to the changes reported in those studies. However, our placebo treated, normal coronary artery segment subgroup showed substantially less vasoconstriction than was observed in the other two studies. This could be explained by the relatively low cardiovascular risk factor profile of our patients compared with those studied by Treasure and Anderson. In particular, our patients had few previous interventions, few were diabetic, there were no current smokers, and not many had had previous cardiovascular events.

Our pravastatin treatment group did not show attenuated vasoconstriction compared with our placebo group. Thus we were not able to demonstrate a significant beneficial effect of pravastatin on coronary endothelial function. This was true for both the dilated and the normal segment subgroups, although in the dilated segments there was a trend in favour of pravastatin. There are two possible reasons for this apparent lack of effect. First, in the context of the low risk profile of the patients in our study, it could be argued that the very small change evoked by acetylcholine in the placebo group led to the differences between the treatment groups being too small to be significant. The second reason might be the relatively short period between PTCA and our assessment of endothelial function. We hypothesised that if endothelial function is important to the process of recovery, it should be restored shortly after PTCA. In retrospect, we might have made our measurements too early in the process of cellular and functional recovery-especially bearing in mind a current view that the recovery process in the vascular wall and lumen may be more prolonged than was previously thought. ${ }^{85}$ In this context the time allowed for the statins to exert their effects might also have been too short. There are studies, however, showing a positive effect on endothelial function within only six weeks. ${ }^{26}$

The studies by Treasure and colleagues and Anderson and associates referred to above reported conflicting results on the ability of 
statins to improve coronary artery endothelial function.

Treasure and colleagues, ${ }^{23}$ in a study of 16 patients with six months of follow up, found a significant difference between lovastatin treated and placebo treated groups. The segments analysed were predefined as having the worst vasoconstrictor reaction at baseline. When the results were averaged over five segments the difference fell to a non-significant level. As in our study, the coronary segments of the statin group remained almost non-reactive over the whole acetylcholine dose range. The recently published CARATS trial (coronary artery reactivity after treatment with simvastatin) by the same group of investigators expanded the initial study. ${ }^{27}$ However, though 60 patients were included in the new trial, the differences between treatment and placebo groups now did not reach significance. ${ }^{27}$

Anderson and associates, ${ }^{24}$ studying 22 patients with 12 months of follow up, failed to show a significant difference between their statin group and their control (diet) group.

In our study, as is to be expected in small scale studies, the non-procedure related event rate was similar between the treatment groups. The procedure related event rate, however, was significantly different: in the placebo group six patients developed serious flow obstructing dissections during dilatation which required stent placement, while no such events occurred in the pravastatin group $(\mathrm{p}<0.05)$. A possible cause for this difference might be the pravastatin induced increase in vascular wall stability demonstrated by Williams and colleagues in $1998 .^{28}$

Current standards of treatment are such that a trial like PREFACE could not be repeated for ethical reasons. This makes the observations of the trial unique, but we are aware that the results cannot be verified.

\section{LIMITATIONS}

Although the vasomotor actions of acetylcholine are thought to reflect endothelial function, strictly speaking the presence of vasoconstriction, as opposed to vasodilatation, yields no direct evidence of endothelial involvement in the changes following PTCA. In common with other investigators, we did not perform synthesis inhibition studies with, for example, L-NMMA to ensure that the vessel changes provoked by acetylcholine were partially endothelium dependent. ${ }^{21232427}$ It was not possible to do this in the setting of a large scale multicentre clinical study.

We did not perform endothelial function tests immediately before and after PTCA, for ethical reasons-acetylcholine might have caused potentially dangerous vasoconstriction in patients undergoing PTCA. However, if we had been able to do this it could have provided useful pretreatment information on the normal segment. It would also have been possible to preselect the normal segment on the grounds of maximum reactivity in order to increase the potential susceptibility to treatment, as has been done in other studies. ${ }^{23}$
In the design of the study we deliberately chose to have a minimal number of confounding risk factors. This might have been to our disadvantage because a study group with more risk factors could have provided more opportunity for endothelial function improvement.

\section{CONCLUSIONS}

In this double blind, randomised, placebo controlled trial, we found a correlation between endothelium dependent vasomotion in normal and dilated coronary artery segments. There was a trend towards a treatment effect of pravastatin.

The PREFACE trial was supported by Bristol-Myers Squibb Co, Princeton, New Jersey, USA.

1 Landau C, Lange RA, Hillis LD. Percutaneous transluminal coronary angioplasty. N Engl f Med 1994;330:981-93.

2 Lefkovits J, Topol EJ. Pharmacological approaches for the prevention of restenosis after percutaneous coronary intervention. Prog Cardiovasc Dis 1997;40:141-58.

3 Mulder HJ, Bal ET, Jukema JW, et al. Pravastatin reduces restenosis two years after percutaneous transluminal coronary angioplasty (REGRESS Trial). Am f Cardiol 2000;86:742-6

4 Bucher HC, Griffith LE, Guyatt GH. Systematic review on the risk and benefit of different cholesterol-lowering interventions. Arterioscler Thromb Vasc Biol 1999;19:187-95.

5 Bertrand ME, McFadden EP, Fruchart JC, et al. Effect of pravastatin on angiographic restenosis after coronary balloon angioplasty. The PREDICT trial investigators. ary angioplasty. f Am Coll Cardiol 1997;30:863-9.

6 Vaughan CJ, Murphy MB, Buckley BM. Statins do more Vaughan CJ, Murphy MB, Buckley BM. Statins do m
than just lower cholesterol. Lancet 1996;348:1079-82.

7 Celermajer DS. Endothelial dysfunction: does it matter? Is it reversible? $\mathcal{F} \mathrm{Am}$ Coll Cardiol 1997;30:325-33.

8 Libby P, Tanaka H. The molecular bases of restenosis. Prog Cardiovasc Dis 1997;40:97-106.

9 Gibbons GH. Endothelial function as a determinant of vascular function and structure: a new therapeutic target. $\mathrm{Am}$ f Cardiol 1997;79:3-8.

10 Pasternak RC, Baughman KL, Fallon JT, et al. Scanning electron microscopy after coronary transluminal angioplasty of normal canine coronary arteries. Am 7 Cardiol 1980;45:591-8.

11 Cartier R, Pearson PJ, Lin PJ, et al. Time course and extent of recovery of endothelium-dependent contractions and relaxations after direct arterial injury. $\mathcal{F}$ Thorac Cardiovasc Surg 1991;102:371-7.

12 Suter TM, Buechi M, Hess OM, et al. Normalization of coronary vasomotion after percutaneous transluminal corononary vasomotion after percutaneous transl
ary angioplasty? Circulation 1992;85:86-92.

13 Weidinger FF, McLenachan JM, Cybulsky MI, et al. Hypercholesterolemia enhances macrophage recruitment and dysfunction of regenerated endothelium after balloon injury of the rabbit iliac artery. Circulation 1991;84:755-67.

14 Walker LN, Bowyer DE. Endothelial healing in the rabbit aorta and the effect of risk factors for atherosclerosis. Hypercholesterolemia. Arteriosclerosis 1984;4:479-88.

15 Sakai A, Hirayama A, Adachi T, et al. Is the presence of hyperlipidemia associated with impairment of endothelium-dependent neointimal relaxation after percutaneous transluminal coronary angioplasty? Heart Vessels 1996;11:255-61.

16 Ludmer PL, Selwyn AP, Shook TL, et al. Paradoxical vasoconstriction induced by acetylcholine in atherosclerotic coronary arteries. N Engl f Med 1986;315:1046-51.

17 Jukema JW, Bruschke AV, van-Boven AJ et al. Effects of lipid lowering by pravastatin on progression and regression of coronary artery disease in symptomatic men with normal coronary artery disease in symptomatic men with normal to moderately elevated serum cholesterol levels. The regression growth evaluation
Circulation 1995;91:2528-40.

18 Faxon DP, Coats W, Currier JW. Remodeling of coronary artery after vascular injury. Prog Cardiovasc Dis 1997;40: $129-40$

19 Hamon M, Bauters C, McFadden EP, et al. Hypersensitivity of human coronary segments to ergonovine 6 months after injury by coronary angioplasty: a quantitative angiographic study in consecutive patients undergoing single-vessel angioplasty. Eur Heart f 1996;17:890-5.

20 Vassanelli C, Menegatti G, Zanolla L, et al. Coronary vasoconstriction in response to acetylcholine after balloon angioplasty: possible role of endothelial dysfunction. Coron Artery Dis 1994;5:979-86.

21 Nishijima $\mathrm{H}$, Meno H, Higashi $\mathrm{H}$, et al. Coronary vasomotor response to acetylcholine late after angioplasty. Fpn Circ 7 1996;60:789-96.

22 Suwaidi JA, Hamasaki S, Higano ST, et al. Long-term follow-up of patients with mild coronary artery disease and endothelial dysfunction. Circulation 2000;101:948-54. 
23 Treasure CB, Klein JL, Weintraub WS, et al. Beneficial effects of cholesterol-lowering therapy on the coronary endothelium in patients with coronary artery disease. $N$ Engl F Med 1995;332:481-7.

24 Anderson TJ, Meredith IT, Yeung AC, et al. The effect of cholesterol-lowering and antioxidant therapy on endothelium-dependent coronary vasomotion. $N$ Engl $\mathcal{f}$ Med 1995;332:488-93.

25 Asakura M, Ueda Y, Nanto S, et al. Remodeling of in-stent neointima, which became thinner and transparent over 3 years: serial angiographic and angioscopic follow-up. Circulation 1998;97:2003-6.
26 Dupuis J, Tardif JC, Cernacek P, et al. Cholesterol reduction rapidly improves endothelial function after acute coronary syndromes. The RECIFE (reduction of cholesterol in ischemia and function of the endothelium) trial. Circulation 1999;99:3227-33.

7 Vita JA, Yeung AC, Winniford M, et al. Effect of cholesterollowering therapy on coronary endothelial vasomotor function in patients with coronary artery disease. Circulation 2000;102:846-51

28 Williams JK, Sukhova GK, Herrington DM, et al. Pravastatin has cholesterol-lowering independent effects on the artery wall of atherosclerotic monkeys. F Am Coll Cardiol 1998;31:684-91.

\section{IMAGES IN CARDIOLOGY}

\section{Coronary sinus diverticulum containing posteroseptal accessory pathway}

A 41 year old male with Wolff-ParkinsonWhite syndrome and symptomatic atrioventricular re-entrant tachycardia was admitted for electrophysiological study. An exceptionally large coronary sinus diverticulum was identified (right) and found to contain the accessory atrioventricular pathway. Successful radiofrequency ablation to this posteroseptal accessory pathway was carried out.

Coronary sinus aneurysms or diverticulae are rare congenital anomalies with only a few published images. Diverticulae associated with a posteroseptal accessory atrioventricular connection were first described in 1985 . They have been identified at necropsy in patients with Wolff-Parkinson-White syndrome or sudden death, during surgical ablation of posteroseptal pathways, and during angiographic and electrophysiological studies. They are venous pouches located within the epicardial layers of the posterior ventricular septum or posterosuperior process of the left ventricle, with necks opening into the proximal coronary sinus.

Surgical series suggest they may be present in $6-9 \%$ of patients with posteroseptal accessory pathways with symptoms severe enough to warrant surgery. In patients undergoing transoesophageal echocardiography following failed attempts at ablation of a

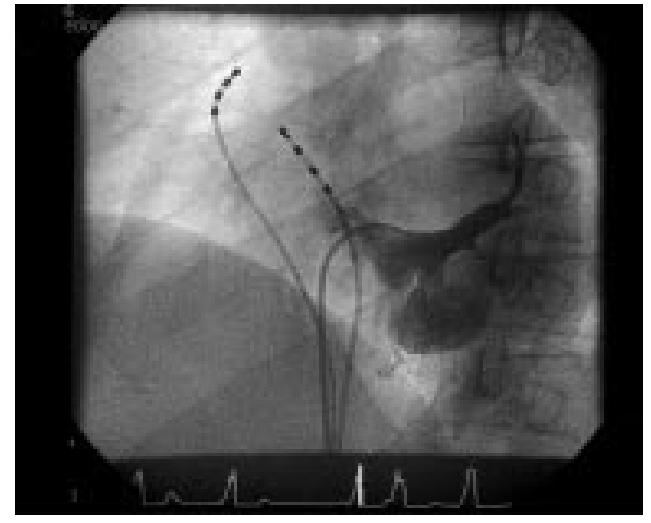

posteroseptal accessory pathway, coronary sinus diverticulae were identified in $13 \%$ of cases.

Coronary sinus diverticulae may be associated with other congenital cardiac abnormalities. Aneurysms of the membranous ventricular septum and subaortic membranes have been described.

Radiofrequency catheter ablation appears to be safe and curative in this setting.

D H BENNETT

M C S HALL mcs.hall@ukgateway.net 